Also, we observed that level of HDLP-C is higher in patients with early stages than in pate stages of $\mathrm{AN}(1,55(1,31-1,57)$ vs. $1,23(1,04-1,36) \mathrm{mmol} / \mathrm{l}, \mathrm{p}=0,04)$. Thus, HDLP-C can be interpreted as a protective factor against contralateral joint involvement in AN patients. The data obtained is consistent with the existing data that HDLP-C lowers the risk for cardiovascular events.

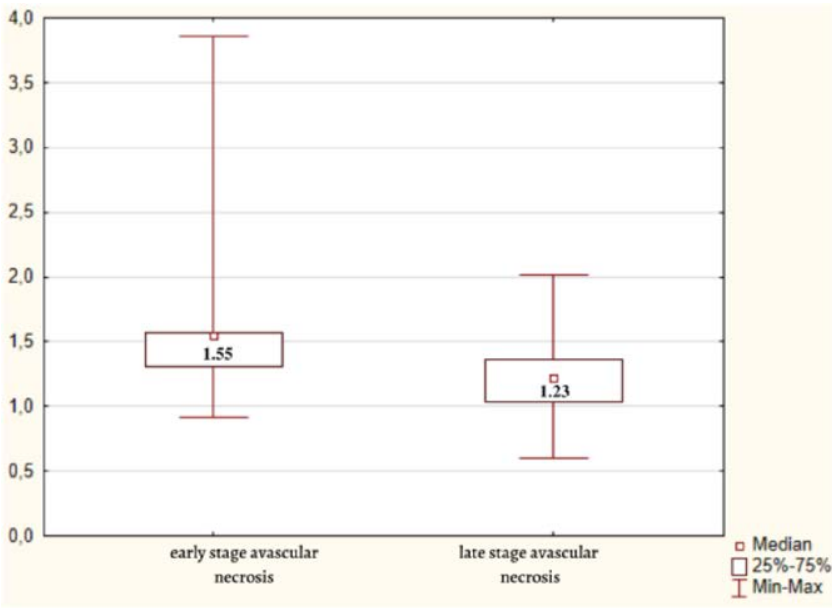

Figure 2. HDLP-C levels in patients with early and late stages of avascular necrosis

Conclusion: The data obtained indicate a significant role of dyslypidemia in the pathogenesis of AN in the context of vascular theory.

References:

[1] Steven G. Chrysant \& George S. Chrysant (2018) The current status of homocysteine as a risk factor for cardiovascular disease: a mini review, Expert Review of Cardiovascular Therapy, 16:8, 559-565, DOI: 10.1080/14779072.2018.1497974.

[2] Narayanan, A., Khanchandani, P., Borkar, R.M. et al. Avascular Necrosis of Femoral Head: A Metabolomic, Biophysical, Biochemical, Electron Microscopic and Histopathological Characterization. Sci Rep 7, 10721 (2017). https://doi.org/10.1038/.

Disclosure of Interests: Katsiarina Gudkevich: None declared, Natalia Martusevich Shareholder of: k, Elena Dashkevich: None declared

DOI: 10.1136/annrheumdis-2020-eular.6505

\section{SAT0522 PROGRESSION OF VISION-RELATED QUALITY OF LIFE AND IDENTIFICATION OF RISK FACTORS IN NON-INFECTIOUS UVEITIS PATIENTS}

I. Hernandez ${ }^{1}$, L. Abasolo ${ }^{2}$, B. Fernandez ${ }^{1}$, A. Madrid García ${ }^{2}$, J. Font ${ }^{1}$, E. Pato ${ }^{1}$, L. Rodriguez Rodriguez ${ }^{2} .^{1}$ Hospital Clínico San Carlos, Rheumatology, Madrid, Spain; ${ }^{2}$ Fundación para la Investigación Biomedica - HCSC, Rheumatology, Madrid, Spain

Background: Uveitis are characterized by inflammation of the middle layer of the eye wall. In developed countries uveitis are the second major treatable cause of blindness in those $20-65$ years of age. Additionally, more than $50 \%$ of the subjects affected with these conditions will develop complications related to the uveitis, and more than $30 \%$ will suffer visual impairment. As a result, these conditions are associated with an important burden. The assessment of the patient's quality of life (QoL) through standardized and validated questionnaires allows us to evaluate objectively the burden of the disease. Several studies have shown that the QoL of uveitis patients is reduced when compared with that of general population. Moreover, several socio-demographic and clinical related characteristics have been associated with impaired QoL. However, no longitudinal analysis of the vision-related (VR) QoL in clinical practice has been carried out.

Objectives: To describe VR-QoL in non-infectious uveitis (NIU) patients during a follow-up period of two years. Furthermore, to analyse the influence of sociodemographic, clinical and treatment factors on the progression of VR-QoL.

Methods: Longitudinal prospective study which includes patients examined in a multidisciplinary tertiary uveitis clinic, with a diagnosis of NIU. In each of these patients a yearly determination of VR-QoL was carried out following the VFQ-25 questionnaire, finally including all those who had completed at least an initial questionnaire and a second one after two years of follow-up. Analysis of risk factors at baseline in repeated VFQ-25 measurements was carried out by generalized estimating equations (GEE) models. Variables related to demographic, clinical and treatment factors with a determination of $\mathrm{p}$-value $<0.15$ were included in multivariable models, which were then compared using the Quasi Akaike Information Criteria (qAIC). A local Ethics Committee approved the execution of this project.

Results: 128 patients were included, 117 of which also had an evaluation after the first year of follow-up. $55.5 \%$ were female with a median age of 34 years at the start of symptoms and of 37 years at the moment of attending our clinic for the first time. First evaluation of VR-QoL was determined a median (p25-p75) of $6.1(1.8-13.1)$ years after that first visit. The most frequent locations of NIU were anterior $(41.1 \%)$, panuveitis $(27.4 \%)$, posterior $(16.1 \%)$ and intermediate $(15.3 \%)$. At our first evaluation, $27.3 \%$ of patients were receiving treatment with topical steroids, $22.3 \%$ oral, $49.2 \%$ immunosuppressant drugs (both synthetic and/or biological) and $19.05 \%$ biological therapies. The median (p25-p75) VFG25 determinations at baseline, first and second years of follow-up were 0.87 (0.78-0.93), $0.88(0.80-0.93)$ y $0.89(0.81-0.94)$, with no significant differences (first year vs. Baseline $p=0.54 ; 2$ years vs. Baseline $p=0.61$ ).

In the GEE multivariable models the presence at baseline of permanent incapacity due to NIU, concomitant thyroid disease, worse visual acuity, unilatera pattern, cataracts, retinal vasculitis, epiretinal membrane and use of azathioprine were independently associated with a worse VR-QoL (Table 1).

Table 1. Risk factors related to VR-QoL in patients with NIU

\begin{tabular}{lll}
\hline Variables & Coef. (IC 95\%) & p-value \\
\hline Visual acuity & $23.6(12.3-34.8)$ & $<0.01$ \\
Permanent incapacity & $-24.8(-33.7$ & $<0.01$ \\
& $-15.9)$ & 0.05 \\
Unilateral NIU & $-2.9(-5.7$ & \\
Cataracts & $-0.006)$ & 0.037 \\
Vasculitis & $-5.2(-10--0.3)$ & 0.011 \\
& $-13.3(-23.4$ & 0.026 \\
Epiretinal membrane & $-3.1)$ & 0.041 \\
Azathioprine & $-6.8(-12.7--0.8)$ & \\
\hline
\end{tabular}

Conclusion: During these two years of follow-up, no significant changes have taken place regarding VR-QoL in patients with NIU assessed at a tertiary centre. Other than visual acuity at baseline, certain ocular manifestations and clinical comorbidities have also been shown to have an independent effect on the VR-QoL of these patients.

Disclosure of Interests: None declared

DOI: 10.1136/annrheumdis-2020-eular.5889

\section{SAT0523 BIOLOGICAL THERAPY IN REFRACTORY ATYPICAL OPTIC NEURITIS. MULTICENTER STUDY}

A. Herrero Morant ${ }^{1}$, C. Álvarez Reguera ${ }^{1}$, V. Calvo del Rio' ${ }^{1}$ O. Maíz Alonso ${ }^{2}$, A. Blanco ${ }^{3}$, J. Narváez ${ }^{4}$, S. Castañeda ${ }^{5}$, E. Vicente ${ }^{5}$, S. Romero-Yuste ${ }^{6}$, R. Demetrio-Pablo ${ }^{7}$, A. Urruticoechea-Arana ${ }^{8}$, J. L. García Serrano ${ }^{9}$, J. L. Callejas Rubio ${ }^{9}$, N. Ortego ${ }^{9}$, J. Sánchez ${ }^{10}$, P. Estrada ${ }^{11}$, I. Rua-Figueroa ${ }^{12}$, D. Martínez-López ${ }^{1}$, J. L. Martín-Varillas ${ }^{1}$, M. Á. González-Gay ${ }^{1}$, R. Blanco ${ }^{1} .{ }^{1} H$. U. M. Valdecilla, Rheumatology, Santander, Spain; ${ }^{2}$ H. Donostia, Rheumatology, Santander, Spain; ${ }^{3} \mathrm{H}$. Donostia, Ophthalmology, San Sebastián, Spain; ${ }^{4} \mathrm{H}$. Bellvitge, Rheumatology, Barcelona, Spain; ${ }^{5} \mathrm{H}$. La princesa, Rheumatology, Madrid, Spain; ${ }^{6}$ H. Pontevedra, Rheumatology, Pontevedra, Spain; ${ }^{7}$ H. U. M. Valdecilla, Ophthalmology, Santander, Spain; ${ }^{8}$ H. Can Misses, Rheumatology, Ibiza, Spain; ${ }^{9} \mathrm{H}$. San Cecilio, Rheumatology, Granada, Spain; ${ }^{10} \mathrm{H} .12$ de Octubre, Rheumatology, Madrid, Spain; ${ }^{11} \mathrm{H}$. Moises Broggi, Rheumatology, Barcelona, Spain; ${ }^{12} H$. Dr. Negrín, Rheumatology, Gran Canaria, Spain

Background: Optic Neuritis $(\mathrm{ON})$ is an inflammation of the optic nerve. Its most common presentation is demyelinating typical ON. Atypical ON is rare, severe, non-demyelinating and can be isolated or associated to different diseases including autoimmune diseases. If it is not treated, it can lead to devastating visual results. Conventional treatment includes systemic corticosteroids and conventional immunosuppressants (CIS).

Objectives: Our aim was to assess the efficacy of biological therapy in atypical ON refractory to conventional treatment.

Methods: Open-label multicenter study including 19 patients diagnosed with atypical ON refractory to systemic corticosteroids and at least one CIS. The main outcomes assessed were Best Corrected Visual Acuity (BCVA) and optic nerve and ganglionar cells Optical Coherence Tomography (OCT). These outcome variables were recorded at baseline, 1 week, 2 weeks, 1 month, 3 months and 6 months and 1 year after biological therapy onset. 